\title{
Correction to: Persistent major alopecia following adjuvant docetaxel for breast cancer: incidence, characteristics, and prevention with scalp cooling
}

M. Martín ${ }^{1,6}$ (1) J. C. de la Torre-Montero ${ }^{2,3} \cdot$ S. López-Tarruella ${ }^{4} \cdot$ K. Pinilla ${ }^{5} \cdot$ A. Casado ${ }^{2} \cdot$ S. Fernandez $z^{2,4} \cdot$ Y. Jerez ${ }^{4}$. J. Puente ${ }^{2} \cdot$ I. Palomero ${ }^{4} \cdot$ R. González del Val $\cdot$ M. del Monte-Millan ${ }^{4} \cdot$ T. Massarrah $^{4} \cdot$ C. Vila ${ }^{2}$ B. García-Paredes ${ }^{2}$. J. A. García-Sáenz $z^{2}$ A. Lluch ${ }^{5}$

Published online: 16 July 2018

(c) Springer Science+Business Media, LLC, part of Springer Nature 2018

Correction to: Breast Cancer Research and Treatment https://doi.org/10.1007/s10549-018-4855-2

In the original publication of the article, Table 1 was published with incorrect caption and values. The Table 1 with corrected caption and values is given in this Correction.

The original article can be found online at https://doi.org/10.1007/ s10549-018-4855-2.

M. Martín

mmartin@geicam.org

1 Medical Oncology Service, Hospital General Universitario Gregorio Marañón, Instituto de Investigación Sanitaria Gregorio Marañón, CIBERONC, GEICAM, Universidad Complutense, Madrid, Spain

2 Medical Oncology Department, Hospital Clínico San Carlos, Instituto de Investigación Sanitaria del Hospital Clínico San Carlos (IdISSC), CIBERONC, Madrid, Spain

3 Universidad Pontificia Comillas, Madrid, Spain

4 Medical Oncology Service, Hospital General Universitario Gregorio Marañón, Instituto de Investigación Sanitaria Gregorio Marañón, CIBERONC, Madrid, Spain

5 Medical Oncology Department, Hospital Clínico Universitario, CIBERONC, Valencia, Spain

6 Hospital General Universitario Gregorio Marañón, Medical Oncology Service, Calle Maiquez, no. 9, 28007 Madrid, Spain 
Table 1 Prevalence study

Number of patients

Median age (years, range)

Menopausal status

Postmenopausal

Premenopausal

Adjuvant therapy

$\mathrm{FAC} / \mathrm{FEC} / \mathrm{AC} / \mathrm{EC} \times 6$ cycles

FAC/FEC/AC/EC $\times 4$ cycles $\rightarrow$ weekly paclitaxel $\times 8-12$ cycles

29

Epirubicin $90 \mathrm{mg} / \mathrm{m}^{2}$ plus docetaxel $75 \mathrm{mg} / \mathrm{m}^{2} \times 4$ cycles $\rightarrow$ Capecitabine $\times 4$ cycles

31

TAC $\times 6$ cycles

74

$\mathrm{A} / \mathrm{E}+/-\mathrm{C} \times 4 \mathrm{cy} \rightarrow$ docetaxel $100 \mathrm{mg} / \mathrm{m}^{2} \times 4$ cycles $+/-$ trastuzumab

66

Tamoxifen ${ }^{\mathrm{a}}$

57

Aromatase inhibitors $^{\mathrm{b}}$

Characteristic of the patients and therapy (HCSC)

HCSC, Hospital Clinico San Carlos, Madrid; FAC: 5-fluorouracil, doxorubicin, cyclophosphamide; FEC, 5-fluorouracil, doxorubicin, cyclophosphamide; AC/EC, doxorubicin or epirubicin plus cyclophosphamide; TC, docetaxel $75 \mathrm{mg} / \mathrm{m}^{2}$ plus cyclophsphamide; TAC, docetaxel $75 \mathrm{mg} / \mathrm{m}^{2}$, doxorubicin, cyclophosphamide; $\mathrm{TCH}$, docetaxel $75 \mathrm{mg} / \mathrm{m}^{2}$, carboplatin AUC 6, trastuzumab

${ }^{a}$ Without prior adjuvant chemotherapy

${ }^{\mathrm{b}}$ Without prior adjuvant chemotherapy; some patients included in this group have received tamoxifen followed by an aromatase inhibitor 